\title{
Effect of the Near Surface Depletion Layer on Photo-Response of Direct Band Gap Semiconductor
}

\author{
Keneni Taye Serdo ${ }^{1} \quad$ Megersa Wodajo Shura ${ }^{2} \quad$ Tamire Legesse Fole $^{3}$ \\ 1.Physics Department, Gambella secondary and preparatory school, Gambella, Ethiopia \\ 2.Physics Department, Adama Science and Technology University (ASTU), Adama, Ethiopia \\ 3.Physics Department, Debark University, Debark, Ethiopia
}

\begin{abstract}
In this study, the effects of the photo-responses of the near surface depletion layer and the deep bulk of gallium antimonide $(\mathrm{GaSb})$ are investigated under different doping levels, injection level and illumination energies. First, the absorption rate of photons is described as a function of illumination energies at different locations inside the sample and as a function of the depth below the illuminated surface for photons with different energies. Then, the doping level dependence of the low injection level radiative, Auger and effective excess carrier lifetimes just under the surface and in the deep bulk are investigated by considering the variation of energy bending at the surface. The variation of the low injection level excess carrier lifetimes with the depth below the illuminated surface for samples with different doping levels is also described. This is followed by the description of the combined effects of doping and illumination energy on the photo-response of the entire bulk at slightly low injection level. Finally, the excess carrier injection level dependence of excess carrier lifetimes under the illuminated surface and deep bulk is also described for samples with different doping levels. Since photon absorption rate is directly related to the free carriers generation rate, the description of the photon absorption rate as functions of illumination energies and the depth below the illuminated surface is found to be the important and factors in the investigation in the photoresponse of semiconductors along with the doping and the injection levels. The analysis of the results also shows that, the under surface region is insensitive to the doping level and that of the deep bulk is highly affected by the doping level. The injection level dependence of the photo-response of the under surface region is similar for all samples with different doping. The energy of illumination each photon mainly suppresses the photo-responses of the points situated closer to the penetration depths of each photon below the illuminated surface of the sample.
\end{abstract}

Keywords: photo-response, direct band gap semiconductor, gallium antimonide $\mathrm{GaSb}$

DOI: $10.7176 /$ APTA/75-03

\section{Introduction}

Semiconductors are materials that are very important in modern optoelectronic technologies. The existence of the energy band gap, high density of charge carrier density in semiconductor makes them very important in this aspect. Almost all of today's technology involves the use of semiconductors, with the most significant aspect being the integrated circuit (IC). Energy band gap is for any semiconductors there is a forbidden energy range in which allowed states cannot exist. There are two types of energy band gap called direct and indirect band gaps. Direct band gaps semiconductors are the materials for which maximum of valence band and minimum of conduction band lie for the same value of wave vector $(\mathrm{K})$. Indirect band gap semiconductors are the materials for which maximum of valence band and minimum of conduction band do not have the same value of wave vector [1-6].

The purpose of this work is in particular to determine the effect of the near surface depletion layer on photoresponse on the photocurrent produced in a direct band gap semiconductor during illumination with a light of photon energy which is greater than the energy band gap of semiconductor. Small direct band gap semiconductors with energy band gap ranged from 0.18 to $1.0 \mathrm{eV}$, like indium arsenide (InAs), gallium antimonide GaSb, indium antimonide ( $\mathrm{InSb}$ ) are characterized by the absence or small amount of impurity materials, presence of very high charge carrier concentrations. Small direct band gap semiconductors find application as detectors in the mediumto-far infrared region [7].

Small band gap materials are used in various domains, such as pollutant detection and infrared thermal imaging, using a variety of device architectures, including near infrared photo-detectors, resonant tunneling structures and other quantum devices. Gallium antimonide ( $\mathrm{GaSb}$ ) and related compounds are direct band-gap semiconductors suitable for fabricating high-frequency electronic devices and optoelectronic devices. The photo response of this material makes it very useful in the design of a variety of device architectures, including photo detectors, resonant tunneling structures and other quantum devices. Some of these semiconductors are also used as booster cells in solar cell clusters to improve the efficiency of photovoltaic and thermo-photovoltaic (TPV) cells. A great deal of civilization hopes rest on continued technological advancement using semiconductors [8].

The radiative generation-recombination and Auger mechanisms are fundamental band-to-band processes which are determined by the electronic band structure of the semiconductor. The role of radiative mechanism in detection of IR radiation has been recently critically re-examined by Humphreys. The Auger mechanism dominates generation and recombination processes in high quality and small band gap semiconductor. Auger recombination 
is typically observed in both direct and indirect band-gap semiconductors when either the doping density or the excess carrier density is very high. Both radiative and Auger recombination can also occur through intermediate energy levels. When electron-hole pairs recombine radiatively through intermediate levels, photons are generally emitted for only one of the transitions [9]. It has thus become necessary to modify older techniques and develop measurement that provide better information specifically about surface and bulk properties.

The performance of the material in this case depends on the lifetime of free charge carriers generated by the interaction of light with the material, referred to as the excess carrier lifetime ( ) Using the results from Hall Effect measurements along with the relations that describe the lifetimes of the excess minority carriers under the surface and in the bulk of the semiconductors, the theoretical values of the effective excess carrier lifetime in the materials were also calculated [10].

Therefore, radiative excess carrier lifetime is constant at low injection, but then decreases and continues to decrease as the injection level increases. While radiative recombination is typically the dominant recombination process in direct band gap semiconductors under low injection level, it is considered to be small or even negligible compared to other recombination processes in indirect semiconductors. Radiation which is presumably due to the recombination of free electrons and holes has been observed in gallium antimonide (GaSb) [11]. The radiative lifetime is inversely proportional to the total concentration of free carriers under all circumstances. Auger recombination will become the dominant mode of recombination direct and in indirect semiconductors for high injection levels. The lifetimes increased with decreased excess carrier density. This is due to effects of Auger recombination and Radiative recombination and the lifetimes were taken at a consistent excess carrier concentration. The lifetime of both radiative and Auger mechanisms decreases with increase in doping concentration and the effective lifetime of the carriers are controlled only by Auger mechanism. Several methods were developed to determine effect of the near surface depletion layer on photo-response of direct band gap semiconductor and contents of carrier concentration in different recombination and lifetime [12-14].

\section{Materials}

\subsection{Energy Band gap}

In a semiconductor, the energy difference between the top of valance band $\left(E_{V}\right)$ and the bottom of conduction band $\left(E_{C}\right)$ is defined as the band-gap energy $\left(E_{g}\right)$. The band-gap energy of a semiconductor defines its optical absorption threshold or beginning for the photoelectric effect. That is, generation of electric charge-carrier from light absorption only occurs with photons with energies greater or equal to the band gap, $E_{g}$ (or $h v \geq E_{g}$ ), where $h$ the Planck's constant and $v$, the frequency of the absorbed photon by the semiconductor. In order to absorb a photon, an electron generally must be excited from the valence band of the semiconductor to the conduction band [16].

The band gap energy $E_{g}=E_{C}-E_{V}$ is perhaps the most important in semiconductor physics. The first empirical relation for the band gap shift with temperature was developed by Varshni et al [17-20]:

$$
E_{g}(T)=E_{g}(0)-\frac{\alpha T^{2}}{\beta+T},
$$

where $\alpha$ and $\beta$ are constants $3.78 \times 10^{-4} \mathrm{eV} / \mathrm{K}$ and $94 \mathrm{~K}$ respectively chosen to obtain the best fit to experimental data and $E_{g}(0)$ is $0.813 \mathrm{eV}$, the limiting value of the band at zero Kelvin. By using Eqns. (2-1) we get energy band gap value which is $\mathrm{GaSb}$ sample $0.726 \mathrm{eV}$ at room temperature. Gallium antimonide $(\mathrm{GaSb})$ is small direct band gap semiconductor and Zinc blend structures [21].

\subsection{Thermal equilibrium charge carriers}

The density of state function in the conduction and valence band of a semiconductor provided that free electron mass either the electrons density of state effective mass $\left(m_{n}^{*}\right)$ in the conduction band or holes density of state effective mass $\left(m_{p}^{*}\right)$ in the valence band.

Effective masses for holes and electrons of states of GaSb are given in [22, 23].

$$
m_{p}^{*}=0.5 m_{0}, \quad m_{n}^{*}=0.047 m_{0} .
$$

The densities of states are determining properties of conduction and valence bands. The density of states in the valence band and conduction band are given as:

$$
\begin{array}{ll}
\rho_{C}(E) & =\frac{4 \pi}{h^{3}} \sqrt{\left(2 m_{n}^{*}\right)^{3}}\left(E-E_{C}\right) \quad \text { and } \\
\rho_{V}(E) & =\frac{4 \pi}{h^{3}} \sqrt{\left(2 m_{p}^{*}\right)^{3}}\left(E_{V}-E\right) .
\end{array}
$$

The total density of electrons in the semiconductor is obtained from Eqns.

(2-3) and Fermi energy as: 


$$
n_{0}=N_{C} \exp \left(-\frac{E_{C}-E_{F}}{k_{B} T}\right) \text {, in the conduction band. }
$$

The total density of holes in the semiconductor is obtained from Eqn.

(2-3) and Fermi energy as:

$$
p_{0}=N_{V} \exp \left(-\frac{E_{F}-E_{V}}{k_{B} T}\right) \text {, in the valence band. }
$$

where, $N_{C}$ and $N_{V}$ the effective density of conduction band states and the effective density of valence band states respectively, which are the expression for the equilibrium holes density in valence band and electrons density in conduction band [24]:

$$
N_{C}=2\left(\frac{2 \pi m_{n}^{*} k_{B} T}{h^{2}}\right)^{1.5}, \quad N_{V}=2\left(\frac{2 \pi m_{p}^{*} k_{B} T}{h^{2}}\right)^{1.5} .
$$

\subsection{Intrinsic Semiconductors}

Intrinsic semiconductors are semiconductors do not contain impurities. A semiconductor may be considered as an intrinsic semiconductor if its thermally generated carrier density represented by $n_{i}$ is much larger than the background doping or residual impurity densities. [25-27] For an intrinsic semiconductor where the Fermi level, is equal to the intrinsic level energy, in the semiconductor from Eqns. (2-4) and (2-5):

$$
n_{i}=N_{C} \exp \left(-\frac{E_{C}-E_{i}}{k_{B} T}\right)=N_{V} \exp \left(-\frac{E_{i}-E_{V}}{k_{B} T}\right),
$$

where intrinsic Fermi energy is given by:

$$
E_{i}=\left(\frac{E_{C}+E_{V}}{2}\right)+\frac{3}{4}\left(k_{B} T\right) \ln \left(\frac{m_{p}^{*}}{m_{n}^{*}}\right) .
$$

Once $m_{p}^{*}, m_{n}^{*}$ and $E_{g}$ are known at a given temperature and one can easily compute the value of $\mathrm{n}_{\mathrm{i}}$ and $\mathrm{E}_{\mathrm{i}}$ using Eqn. (2-8) into (2-7). In the non-degenerate semiconductor case:

$$
n_{i}=\sqrt{n_{0} p_{0}}=\sqrt{N_{C} N_{V}} \exp \left(-\frac{E_{g}}{2 k_{B} T}\right) \text {. }
$$

Eqn. (2-9) is called law of mass action. Which indicates that if $m_{p}^{*}=m_{n}^{*}$ or $T=0$ the Fermi level in an intrinsic semiconductor is positioned at mid-gap [28, 29].

\subsection{Extrinsic semiconductors}

In an extrinsic semiconductor, electrons are majority carriers and holes are minority carriers in n-type semiconductor. In p-type material, electrons are minority carriers and holes are majority carriers. Adding donor or acceptor impurity atoms into semiconductor will change the distribution of electrons and holes in the material, and as a result, the Fermi energy will change and eliminating $N_{C}$ and $N_{V}$ among Eqns.(2-4), (2-5) and (2-7) yields [30]:

$$
n_{0}=n_{i} \exp \left(-\frac{E_{i}-E_{F}}{k_{B} T}, \quad p_{0}=n_{i} \exp \left(-\frac{E_{F}-E_{i}}{k_{B} T}\right)\right. \text {. }
$$

The law of mass action Eqn. (2-9) allows one to calculate the minority carrier density in an extrinsic semiconductor when the majority carrier density is known:

$$
\text { Minority carrier density }= \begin{cases}\frac{n_{i}^{2}}{p_{0}}, & \text { for } p \text { type material, } \\ \frac{n_{i}^{2}}{n_{0}}, & \text { for } n \text { type material. }\end{cases}
$$

The thermal equilibrium extrinsic Fermi-level position can also be described from Eqn.(2-10) in terms of $E_{i}$ and the thermal equilibrium carrier concentrations as: [31]

$$
E_{F}=E_{i}-k_{B} T \ln \left(\frac{p_{0}}{n_{i}}\right)=E_{i}+k_{B} T \ln \left(\frac{n_{0}}{n_{i}}\right) .
$$

As the charge carrier concentrations change, the position of the extrinsic Fermi energy level varies within the band gap until the system reaches thermal equilibrium Eqns. (2-12) shows that $E_{F}$ lies above $E_{i}$ for n-type material $\left(n_{i}>p_{0}\right)$ and below $E_{i}$ for p-type material $\left(n_{i}<p_{0}\right)[32-33]$.

\subsection{Statistics of Donors and Acceptors}

The density of electrons in a shallow-donor state is given by [34];

$$
n_{d}=\frac{N_{d}}{1+\frac{1}{g_{d}} \exp \left(\frac{E_{d}-E_{F}}{k_{B} T}\right)},
$$


where, $n_{d}$ the density of electrons occupying the donor level is, $N_{d}$ is the concentration of donor atoms, $E_{d}$ is the energy of the donor level and $g_{d}$ is the donor-site degeneracy factor. The concentration of ionized donors $N_{d}^{+}$is then:

$$
N_{d}^{+}=N_{d}-n_{d}=\frac{N_{d}}{1+g_{d} \exp \left(\frac{E_{F}-E_{d}}{k_{B} T}\right)} .
$$

When a singly-ions able donor captures an electron it becomes neutral, and when it releases an electron, it becomes positively charged. Using the same analysis for the concentration of ionized acceptors $N_{a}^{-}$we have:

$$
N_{a}^{-}=N_{a}-p_{a}=\frac{N_{a}}{1+g_{a} \exp \left(\frac{E_{a}-E_{F}}{k_{B} T}\right)},
$$

where $p_{a}$ is the density of holes bound to acceptors, $N_{a}$ the concentration of acceptor atoms, $E_{a}$ the energy of the acceptor level relative to the valence band, $N_{a}^{-}$the concentration of ionized acceptor atoms and $g_{a}$ is the acceptorsite degeneracy factor. When free electron-hole pairs annihilate through recombination, the free carrier concentrations in the conduction and valence bands adjust for the intrinsic semiconductor to $n_{0}=p_{0}=n_{i}$. For the extrinsic semiconductors this takes the form:

$$
n_{0}=N_{d}^{+}+\mathfrak{N}-n_{R}, \quad p_{0}=N_{a}^{-}+\mathfrak{N}-n_{R},
$$

where $n_{R}$ is the concentration of the total electron-hole pairs being annihilated each other after the donors and the acceptors are being ionized, $\mathfrak{N}$ is the concentration of electron-hole pairs thermally generated from band to band and $N_{a}^{-}$and $N_{d}^{+}$are the densities of free electrons and holes contributed by the donor and the acceptor levels to the respective conduction and valence bands. Upon eliminating $\mathfrak{N}-n_{R}$ in Eqn. (2-16) the well-known neutrality relationship is obtained:

$$
N_{d}^{+}+P_{0}=N_{a}^{-}+n_{0} \text {. }
$$

The law of mass action Eqn. (2-9) along with Eqn. (2-17) yields:

$$
p_{0}=\frac{N_{a}^{-}-N_{d}^{+}}{2}+\sqrt{n_{i}^{2}+\left(\frac{N_{a}^{-}-N_{d}^{+}}{2}\right)^{2}} \text {. }
$$

For very high acceptor impurity concentrations $\left(N_{a}^{-} \gg N_{d}^{+}\right)$, Eqn. (2-18) can be simplified:

$$
P_{0}=N_{a}^{-}+n_{i}
$$

\subsection{The near surface depletion region at thermal equilibrium}

In the depletion region of a semiconductor, the free carrier concentration is very small as compared to the bulk, also called depletion region, as well as junction region or space charge region is an insulating region within a conductive or doped semiconductor layer where the charge carriers have been swept away through recombination $[35,36]$.

In the depletion layer the majority carrier concentration at the surface is smaller than its equilibrium value, but larger than the minority carrier concentration at the surface, and the bulk of most isolated III-V semiconductors have three various regions of different carrier concentrations. This consists of a neutral region (with high thermal equilibrium majority carrier concentration), followed by a transition layer (with decreasing thermal equilibrium majority carrier concentration in moving towards the surface), and a depleted surface region of very low thermal equilibrium majority carrier concentration. The width of the total depletion layer is represented by $b_{1}$ [37-39].

The band bending in different layers accounts for the variation of the majority carrier concentration in these layers. The description of the band bending, $E_{S}(x)$ at any location $x$ in the depletion region also enables us to evaluate the thermal equilibrium free carriers densities at any point in the depletion region. The majority carrier density, $p(E)$ at any energy, $E$ in the depletion layer of a $p$-type semiconductor can be expressed as:

$$
p(E)=p_{0} \exp \left(\frac{-E}{k_{B} T}\right) \text {. }
$$

The majority carrier concentration in Eqn. (2-20) is in general decreases with increasing the band bending. The minority carrier concentration at any point in the depletion layer can be described using the law of mass action Eqn.(2-9) along with Eqn. (2-20) as:

$$
n(E)=\frac{n_{i}^{2}}{p(E)}=n_{0} \exp \left(\frac{E}{k_{B} T}\right) \text {. }
$$

The width of the total depletion region $b_{1}$ also can be rewritten as:

$$
b_{1}=\sqrt{\frac{2 \epsilon E_{s}}{e^{2} N_{a}^{-}}} .
$$

where $\epsilon$ is the permittivity of the material. The position dependence of the bending in energy in the entire depletion 
layer between the surface and the edge of the quasi-neutral region can be described as:

$$
E(x)=-\frac{e^{2} N_{a}^{-}}{2 \epsilon}\left(b_{1}-x\right)^{2} .
$$

Where $x$ is depth at surface. Figure 1 illustrates the position dependence of (a) energy band structure and (b) thermal equilibrium majority carrier concentration in the under surface depletion layer and the deep bulk of p-type semiconductor. As can be seen from Figure 1 (b), the majority carrier concentration decreases from the deep bulk to the surface and hence the minority carrier concentration increases from the deep bulk to the surface. The energy band bending also increases from the deep bulk to the surface (see Figure 1 (a)).
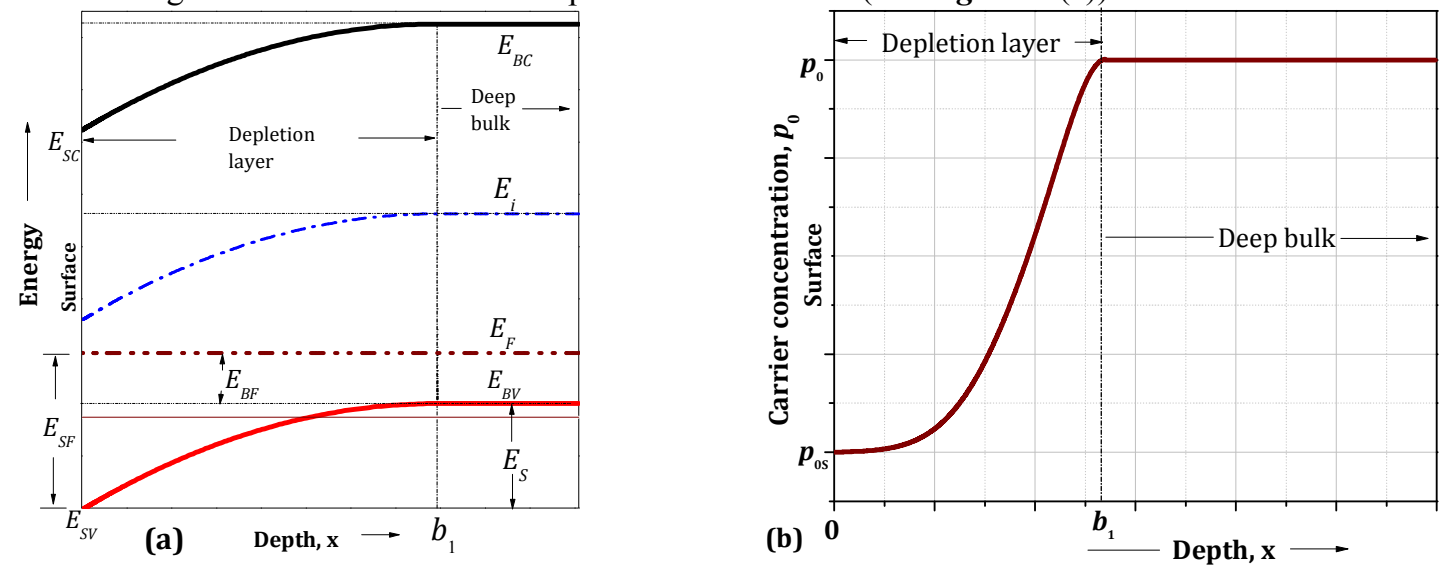

Figure 1: Position dependence of (a) energy band structure and (b) thermal equilibrium majority carrier concentration in the under surface depletion layer and the deep bulk of p-type semiconductor.

The valence band maximum (VBM) in the neutral region (bulk) is represented by $E_{B V}$ and that at the surface is represented by $E_{S V}$ respectively. The respective conduction band minimum (CBM) in the neutral region (bulk) and at the surface are represented by $E_{B C}$ and $E_{S C}$ respectively. The total band bending at the surface is represented by $E_{S}$. The Fermi-level position relative to the VBM is denoted by $E_{B F}$ in the bulk and by $E_{S F}$ at the surface. The intrinsic energy level in the entire sample is also represented by $E_{i}$. In the entire depletion layer the energy bends down and reaches its maximum at the surface. The majority carrier concentration in the depletion layer also decreases from maximum value $p_{0}$ at the edge of the quasi-neutral region toward to the surface and attains its minimum value $p_{0 S}$ in the entire region between the edge of the transition region and the surface [40].

The total width of depletion layer relative to the surface and in the bulk at room temperature for highly doped p-type semiconductor. The total width of depletion layer including transition region and highly depleted region. Majority concentration and minority carrier concentration in the bulk is constant until the total depletion width and after this because of band bending energy at the surface majority carrier concentration decreases with increasing minority carrier concentration under the surface [40-42].

The thermal equilibrium majority carrier density $p_{0 S}$ under the surface of a p-type semiconductor can also be expressed in terms of the surface Fermi-level position $E_{S F}$, using Eqn. (2-20) as:

$$
p_{0 S}=p_{0} \exp \left(-\frac{E_{S}}{k_{B} T}\right)=n_{i} \exp \left(\frac{E_{i}-E_{S F}}{k_{B} T}\right) \text {. }
$$

Since, $n_{i}$ and $E_{i}$ in Eqns. (2-24) are not the function of $N_{a}$, the doping level dependence of $p_{0 S}$ can be described only if the doping level dependence of the surface Fermi-level position, $E_{S F}$ is known. Equation that relates the band bending and the Fermi-level position at the surface of a $\mathrm{p}$ - type semiconductor is:

$$
E_{S}=E_{S F}-E_{B F} \text {. }
$$

Fermi energy at the bulk is given by:

$$
E_{B F}=E_{i}-k_{B} T \ln \left(\frac{p_{0}}{n_{i}}\right)
$$

Figure 2 illustrates the room temperature doping level dependences of (a) the depletion width $\left(b_{1}\right)$, (b) energy band bending at the surface $\left(E_{S}\right)$ and surface Fermi level position $\left(E_{S F}\right)$ in GaSb semiconductor. 

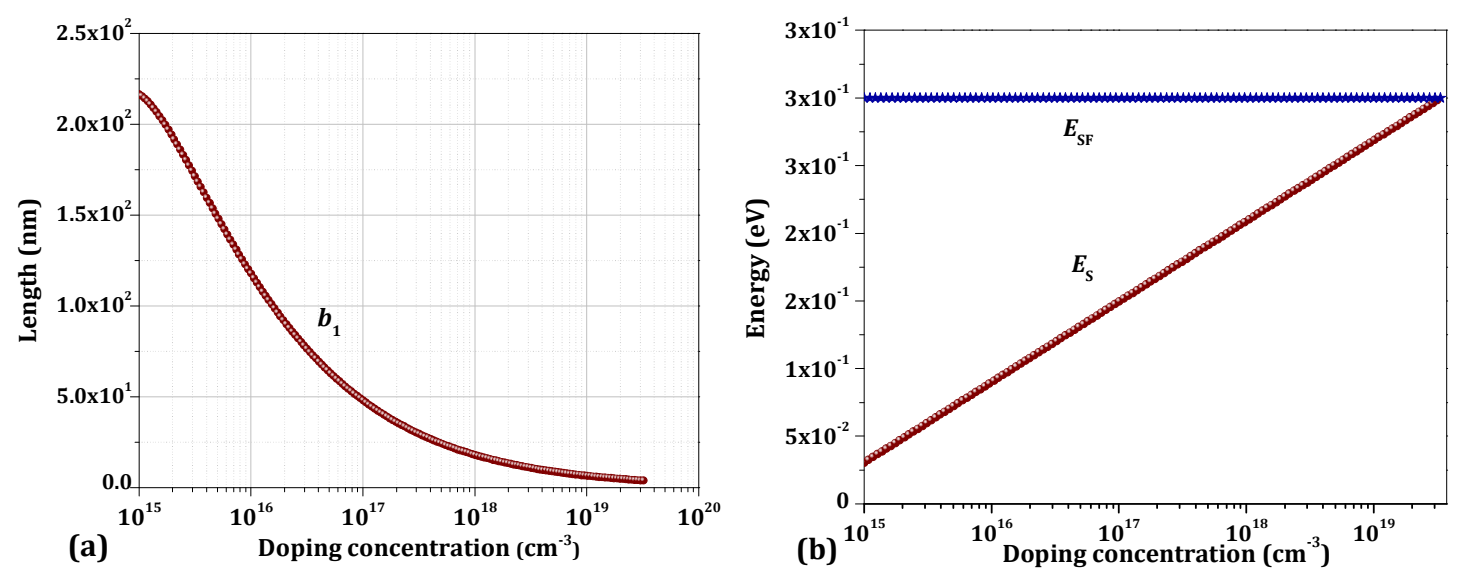

Figure 2: Room temperature doping level dependences of (a) the depletion width $\left(b_{1}\right)$, (b) energy band bending at the surface $\left(E_{\mathrm{S}}\right)$ and surface Fermi level position $\left(E_{\mathrm{SF}}\right)$ in GaSb semiconductor.

As can be seen from Fig. 2(a), the width of the under surface depletion layer decreases with increasing the doping level. The Fermi level position at the surface relative the valence band at the surface, $E_{S F}$ remains constant with increasing the doping level. The energy band bending at the surface increases with increasing the doping level as depicted in Fig. 2(a). These variations bring the difference between the photo-responses of the under surface depletion layer and the deep bulk of a semiconductor.

\subsection{Photo response of Semiconductors}

The interaction of light with matter can be viewed macroscopically as consisting of four components: incident, reflected, transmitted and absorbed (or scattered) components. Using the reflected, the transmitted and the absorbed (or scattered) components of photons one can describe several properties of the material. Reflectance can be defined in terms of the index of refraction of the media on either side of the surfaces (interfaces).

\subsubsection{Optical absorption and carriers Generation in semiconductor}

To select a design of direct small band gap semiconductor sample is gallium antimonite (GaSb) with a model known as length, width and infinitely large thickness denoted by $\ell, w$ and $b$, respectively. The practical magnitudes of the dimensions of the samples are micrometers to be compatible with the magnitudes of the parameters of the illuminating radiations. The beam contains only photons of energy $\hbar v \geq \mathrm{E}_{\mathrm{g}}$ selected by a monochromatic. Figure 3 describes the absorption of photon in bulk semiconductor. As can be seen from Figure, a beam of photon of flux density, $\Phi_{0}$ is directed at the upper surface of a portion of a semiconductor sample [43].

The photon absorption rate $g_{o}$ in the entire volume of the given illuminated area $A$ and thickness $\Delta x$ is defined as the total number of absorbed photons per unit volume per unit time:

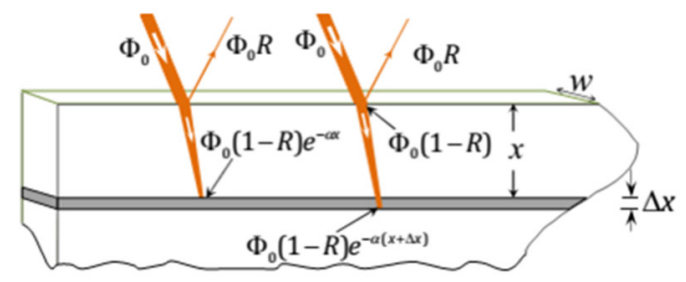

Figure 3: Absorption of photons in bulk semiconductor

$$
g_{o}=\frac{\Delta N_{p h}}{A \Delta x \Delta t}=\frac{\Delta \Phi}{\Delta x},
$$

where $N_{p h}$ is the number of absorbed photons, $\Delta t$ the time and $\Delta \Phi$ is the absorbed photon flux density given by:

$$
\Delta \Phi=\frac{\Delta N_{p h}}{A \Delta t}
$$

As the beam passes through the sample, the photon flux density at a distance $x$ from the surface can be calculated by considering the probability of absorption within any infinitesimal thickness $d x$. The photon absorption rate at depth $x$ for a given wavelength is also proportional to the photon flux density, $\Phi$ remaining at $x$ after being absorbed in the preceding section of material:

$$
g_{o}(x)=\frac{d \Phi(x)}{d x}=-\alpha \Phi(x),
$$

where $\alpha$ is a constant called the absorption coefficient, which is related to the dielectric properties of the material and the wavelength of the incident photon, as will be seen later. The negative (-) sign in Eqns.(2-29) indicates the decrease of $g_{o}$ with increasing $x$. the value of the absorption coefficient determined using its dependence on the 
photon energy.

$$
\alpha(\omega)=\frac{2^{\frac{2}{3}} m_{n} e^{2}}{3 \hbar^{2} \sqrt{k \infty}}\left(\frac{m_{n}^{*} m_{p}^{*}}{m_{n}\left(m_{n}^{*}+m_{p}^{*}\right)}\right)^{\frac{3}{2}}\left(1+\frac{m_{n}}{m_{n}^{*}}+\frac{m_{n}}{m_{p}^{*}}\right)\left(\frac{\hbar \omega-E_{g}}{m_{n} c^{2}}\right) \frac{1}{2},
$$

where $\hbar$ is $h / 2 \pi$ the reduced Plank's constant, $e$ is the elementary charge, $c$ the speed of light and $m_{n / p}$ is the effective electron/hole mass. This allows $\alpha(\omega)$ to be calculated for all wavelengths of the incident photons. Taking into account the reflection of a photon flux density $\Phi_{0} R$ back to the vacuum or air, the photon flux density entering the illuminated surface is given by the difference between the incident and the reflected photon flux densities:

$$
\Phi_{S}(0)=\Phi_{0}(1-R) \text {. }
$$

The solution for Eqn. (2-29) at a distance $x$ below the illuminated surface, using the boundary condition in Eqn. $(2-31)$ results in:

$$
\Phi(x)=\Phi_{0}(1-R) e^{-\alpha x} .
$$

Equation (2-32) is known as Beer's law. It represents the total photon flux density as a function of distance $x$ below the illuminated surface. The reflection coefficient (reflectivity) $R$ is calculated assuming that the refractive index $n$ remains constant for the entire wavelength range under consideration, i.e. $\lambda<20 \mu \mathrm{m}$. Using this assumption, $R$ for a beam incident normal to the surface is given by:

$$
R=\left(\frac{n-1}{n+1}\right)^{2}
$$

For GaSb, the refractive index was taken as 3.8. This results in a typical value of $R \approx 0.34$. The photon flux density absorbed in the elemental thickness $\delta x$ at a distance $x$ below the illuminated surface can be described from Eqns. (2-29) and (2-32) as:

$$
\delta \Phi=\alpha \Phi_{0}(1-R) e^{-\alpha x} \delta x .
$$

Eqns. (2-27) and (2-34) yield the photon absorption rate for different wavelengths as:

$$
g_{0}(\alpha, x)=\alpha \Phi_{0}(1-R) e^{-\alpha x} .
$$

The photon absorption rate in Eqn. (2-35) also depends on the absorption coefficient, $\alpha$. The linear term dominates for lower values of $\alpha$, while the exponential term dominates for higher values of $\alpha$. It has a maximum value for intermediate values of, $\alpha$ which can be found by differentiating Eqn. (2-35) with respect to $\alpha$ and equating it to 0 :

$$
\frac{d g_{0}(\alpha, x)}{d x}=(1-\alpha x) \Phi_{0}(1-R) e^{-\alpha x}=(1-\alpha x) \Phi(x) .
$$

The photon flux density $\Phi(x)$ cannot be zero at all points inside the materials and therefore $(1-\alpha x)=0$ or $\alpha=1 / x$. This defines a depth $x$ below the illuminated surface where the maximum absorption rate occurs for a specific absorption coefficient (i.e. photon wavelength). The depth $x$ at which $g_{o}(\alpha, x)$, has a maximum value for a given photon energy is called the penetration depth $\left(x_{p}\right)$.

The photo-generated electrons and holes due to absorption of photons can change the total electron and hole concentrations in the semiconductor to new values $n$ and $p$, respectively, given by:

$$
n=n_{0}+\delta n \quad \text { and } \quad p=p_{0}+\delta p \text {, }
$$

where $n_{0}$ and $p_{0}$ are the thermal equilibrium electron and hole concentrations; $\delta \mathrm{n}$ and $\delta p$ are excess (photogenerated) electron and hole concentrations [44].

\subsubsection{Excess Carriers recombination in a semiconductor}

Recombination refers to any process whereby any electron is captured by a free hole and both of them stopped the conduction process. Electron has not been vanished in the process of recombination. Depending on the ways in which the energy of an excess carrier is regained during a recombination process, there are three basic recombination mechanisms that are responsible for carriers' annihilation in a semiconductor. They are (1) nonradiative recombination (i.e., the multi-phonon process), (2) band-to-band radiative recombination, and (3) Auger band-to-band recombination $[45,46]$.

When there is generation of charge carriers, it is always balanced at steady-state by the recombination of charge carriers. Recombination of charge carriers takes place both in the bulk and at the surface of a semiconductor. In deriving our recombination model, it has been assumed that the semiconductor is non-degenerate [47- 51].

The rates of change of electrons in the conduction band and holes in the valence band are equal and always given by the difference between the generation and the recombination rates i.e.

$$
\frac{d n}{d t}=\frac{d p}{d t}=G-R,
$$

where $n$ and $p$ represent the non-thermal equilibrium electron and hole concentrations in the conduction and valence bands, respectively, $G$ and $R$ are the total generation and recombination rates of charge carriers, respectively. 
The net excess carrier recombination rate $U$ is defined as the difference between the total recombination rate and the thermal equilibrium generation rate:

$$
U=R-R_{t h},
$$

where $R$ is non-thermal equilibrium recombination, $R_{t h}$ thermal equilibrium recombination. The net recombination rates for photo-generated electrons and holes are given by:

$$
U=\frac{\delta n}{\tau_{n}}=\frac{\delta p}{\tau_{p}},
$$

where $\delta n$ and $\delta p$ are the concentrations of photo-generated carriers given in Eqns. (2-37) the parameters $\tau_{n}$ and $\tau_{p}$ are called the excess electrons and holes recombination lifetimes, respectively. Using the principle of detailed balance $\left(R_{t h}=G_{t h}\right)$, along with Eqns. (2-38) to (2-40) the rates of change of the excess carriers are:

$$
\frac{d \delta n}{d t}=G_{0}-U=\frac{d \delta p}{d t} .
$$

Electron-holes pairs are continuously generated under illumination and the excess charge carrier's concentration will increases. Recombination, on the other hand, tries to reduce the excess carrier concentrations to zero. At steady state, when the recombination rate equals the generation rate:

$$
\delta n=G_{0} \tau_{n} \text {. }
$$

In a semiconductor the term bulk refers to a region in the base material which is free of valence and conduction band bending, typically associated with a disturbance in the periodicity of crystalline structure, such a disturbance happens near a surface.

\section{Radiative recombination Mechanism}

The radiative recombination is the inverse process of fundamental optical absorption in a semiconductor. The recombination of an electron and a hole take place with the release of energy which is equal to the initial difference in the energies of the two charged particles. The released energy is given out in the form of radiation [52].

In non-degenerate semiconductor a photon with energy $h v$ enters a semiconductor transmitted, if $h v<E g$, absorbed if $h v>E g$. Critical photon absorption energy can be used to determine $E g$ of semiconductors. If a photon is absorbed by the semiconductor an electrons holes pair is generated $h v>E g$. Then electrons can be promoted to energy levels above the bottom of the CB. Such electrons lose energy to the lattice until their average velocity approaches the equilibrium velocity of the electrons in the $\mathrm{CB}$. In thermal equilibrium, the rate of recombination is equal to the rate of thermal generation, which can be expressed by:

$$
R_{0}=C_{R} p_{0} n_{0}=C_{R} n_{i}^{2}
$$

where $C_{R}$ is the rate of radiative capture probability derived from the optical absorption process by using the principles of detailed balance.

Under the steady-state conditions, the rate of radiative recombination is given by:

$$
R=C_{R} p n \text {. }
$$

The net recombination rate is obtained by solving Eqns. (2-43) and (2-44) result yields:

$$
U=R-R_{0}=C_{r} \delta n\left(p_{0}+n_{0}+\delta n\right) \text {. }
$$

The radiative lifetime, $\tau_{R}$ due to the recombination is obtained by solving Eqns. (2-40) and (2-45) which yields:

where

$$
\tau_{R}=\frac{\delta n}{U}=\frac{1}{C_{R}\left[n_{0}+p_{0}+\delta n\right]}=\frac{\tau_{R 0}}{1+C_{R} \tau_{R 0} \delta n} .
$$

$$
\tau_{R 0}=\frac{1}{C_{R}\left(n_{0}+p_{0}\right)} .
$$

From Eqn. (2-46), it is noted that $\tau_{R}$ is inversely proportional to the majority carrier density $p_{0}$. Upon substituting the steady-state generation relation (2-42) into Eqn. (2-46), the radiative excess carrier lifetime takes the form:

$$
\tau_{R}=\frac{2 \tau_{k} \tau_{R 0}}{\tau_{R 0}+\tau_{k}}
$$

where $\tau_{k}$ is the time constant given by:

$$
\tau_{k}=\frac{\tau_{R 0}}{\sqrt{1+4 C_{R} G_{0} \tau_{R 0}^{2}}} .
$$

Under low injection conditions, $\delta n \ll p_{0}, n_{0}$, Eqn. (2-46) can be simplified to:

$$
\tau_{R}=\tau_{k}=\tau_{R 0} \text {. }
$$

This shows that, for the low injection case, the radiative excess carrier lifetime is dominated only by the thermal equilibrium majority carrier density [53].

\section{Auger recombination Mechanism}

Auger recombination is a three-particle process, which involves either the electron-electron collisions in the conduction band followed by recombination with holes in the valence band, or hole-hole collisions in the valence 
band followed by recombination with electrons in the conduction band. For small band gap semiconductors such as InSb , GaSb the minority carrier lifetime is usually controlled by Auger recombination, and energy loss is carried out either by electron-electron collisions or hole-hole collisions and subsequent Auger recombination. The Auger recombination lifetime for excess carriers are then given by [54]:

$$
\begin{gathered}
\tau_{A}=\frac{\tau_{A 0}}{1+\tau_{A 0}\left(k_{A} \delta n+C_{A} \delta n^{2}\right),} \\
\text { where } k_{A}=C_{p a}\left(2 p_{0}+n_{0}\right)+C_{n a}\left(p_{0}+2 n_{0}\right), \\
C_{A}=C_{p a}+C_{n a} \quad \text { and } \\
\tau_{A 0}=\left(\frac{1}{C_{p a}\left(p_{0}^{2}+2 n_{i}^{2}\right)+C_{n a}\left(n_{0}^{2}+2 n_{i}^{2}\right)}\right) .
\end{gathered}
$$

Under low injection level conditions $\left(\delta n=\delta p \ll p_{0}, n_{0}\right)$ :

$$
\tau_{A}=\tau_{A 0} .
$$

\section{Effective Excess carrier's lifetime}

The concept of lifetime is introduced here to estimate the time span of life of the carrier before its death by recombination. It is a measure of the period of recovery of carriers from the perturbed state to the equilibrium one [55]. The excess carrier lifetime in an extrinsic semiconductor is dominated by the minority carrier lifetime. The minority carrier lifetime is a key physical parameter for determining the excess carrier recombination in an extrinsic semiconductor under low injection conditions [56, 57].

The effective excess minority carrier lifetime in the entire sample (i.e. bulk plus surface recombination) is determined by considering the sum of all the recombination rates through all the recombination channels. This is the sum of the bulk recombination rate and the surface excess minority carrier recombination rate [58]. The recombination lifetime in the bulk determined by the recombination's mechanism radiative and Auger recombination according to the relationship (2-46) and (2-51):

$$
\frac{1}{\tau_{e f f}}=\frac{1}{\tau_{R}}+\frac{1}{\tau_{A}}
$$

\section{Methodology}

For the analysis of the photo-response of the materials in this work, Gallium antimonide (GaSb) was found to be suitable for its small and direct band gap, very high carrier densities, mobility and its very high purity. The majority carrier concentration, $p_{0}$ used in the analysis for different doping levels GaSb samples was taken from the simulation of the existing temperature dependence Hall measurements data measured at Nelson Mandela Metropolitan University, South Africa. The minority carrier concentration, $n_{0}$ is determined from $p_{0}$ and the intrinsic carrier concentration using the law of mass action for a particular semiconductor. The typical carrier capture coefficients used in the analysis were $2.3 \times 10^{-11} \mathrm{~cm}^{-3} \mathrm{~s}^{-1}$ for radiative recombination, $2.5 \times 10^{-26} \mathrm{~cm}^{6} \mathrm{~s}^{-}$ ${ }^{1}$ for Auger holes capture and $2 \times 10^{-29} \mathrm{~cm}^{6} \mathrm{~s}^{-1}$ for Auger electron capture.

Then, the photon absorption rate in the entire semiconductor is described using the technique discussed in section 2.7.1. The model of bulk semiconductor most used was illustrated in Figure. By using illumination mechanisms the total absorbed photon flux density, the penetration depth $\left(x_{p}\right)$ of a photon in a semiconductor, the photon absorption rate at the surface and in the bulk of a very thick semiconductors were studied in details and the photon absorption rate at a point at depth $x$ below the illuminated surface in very thick semiconductor were determined from the relation shown in eqn. (2-35). By using illumination mechanism, the total photon flux density and photon absorption rate in a layer of thickness $\Delta x$ below the illuminated surface were investigated in details. Moreover, the photon absorption rate at a point situated $x$ distance below the illuminated surface was described. In the analysis of this result, the vertical axis for the photon absorption rate and horizontal axis of the absorption coefficient and depth below the surface was taken as semi-log for both thin film and thick film semiconductor.

By using the steady-state radiative recombination mechanism for small band gap of a p-type semiconductor, the effective excess carrier lifetime was determined using the two recombination mechanisms radiative and Auger that are usually dominant in small band gap materials. The radiative $\left(\tau_{R}\right)$ and Auger $\left(\tau_{A}\right)$ lifetimes for excess carriers in p-type GaSb material are determined as functions of doping, depth below the surface and injection levels. First, the data for energy bending and radiative excess carrier lifetime, $\tau_{\mathrm{R}}$ was described using Eqns. (2-23) and (2-50) in the bulk as function of the depth below the surface for samples of different doping levels. The results of the analyses were described using semi-log graphs for all the excess carrier lifetimes. The results of the doping level dependence at room temperature effective carrier lifetime $\left(\tau_{\text {eff }}\right)$ were described using the combinations of Eqns.(50),(2-53) and (2-54) under the illuminated surface and in the bulk sample of gallium antimonide by varying the doping levels in both the surface and the bulk regions. To describe the photo-response at different depths below the illuminated surface in the semiconductor, the doping level and the absorption coefficient dependence of the radiative excess minority carrier lifetimes are described at different depths in the depletion layer 
and in the deep bulk of the semiconductor.

To corroborate the effects of injection level in all the analysis methods performed under different situations discussed above, both the radiative and the Auger excess minority carrier lifetimes are determined as a function of the optically generated free carriers just under the surface and in the deep bulk for samples of semiconductors with different doping levels.

The effects of the doping level, the energy and the injection level of the illumination on the photo-response of the under surface depletion layer and the deep bulk of a given semiconductor is discussed in detail in the next topic.

\section{Results and discussion}

This topic deals with the analysis and discussion of the entire results of this paper. First, the photon absorption effects at different positions in a semiconductor were determined. Then, the photo-response (excess carrier lifetimes) for both in the deep bulk and the near surface depletion layer in semiconductors were analyzed.

\subsection{Photon absorption rate in a semiconductor}

Figure 4 depicts the ratio of the optical absorption rate per incident photon flux density $\left(g_{0} / \Phi_{0}\right)$ in the bulk of semiconductors as (a) a function of absorption coefficient at different depths in the material and (b) as a function of depth for photons with different absorption coefficients. The graphs of photon absorption rate in Figure 4 (a) and (b) were obtained using relation (2-35).

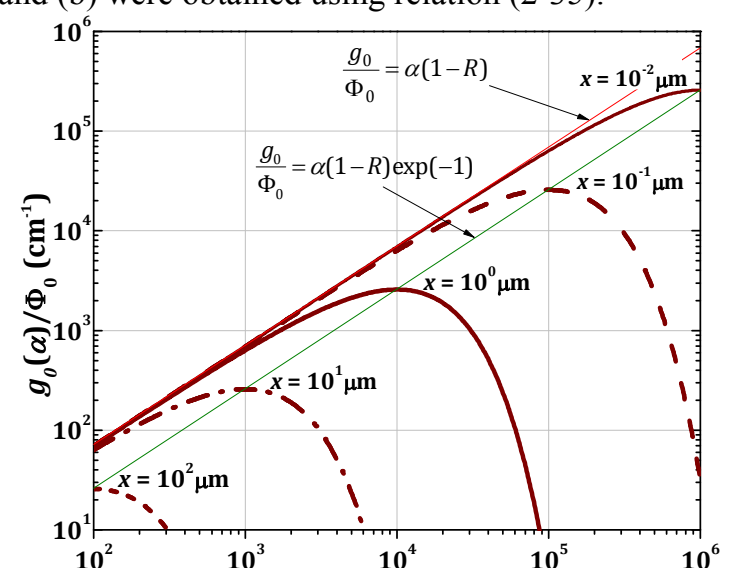

(a)

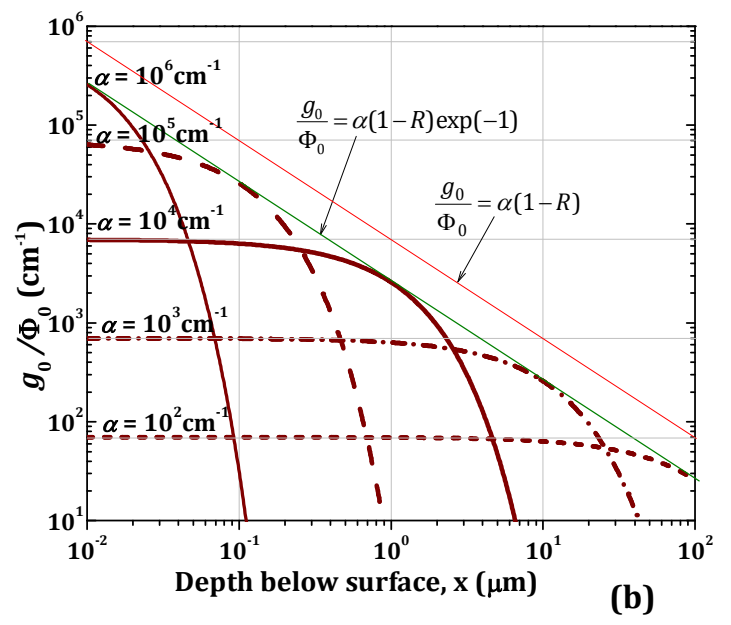

incident photon flux density $\left(g_{0} / \Phi_{0}\right)$ semiconductors depths in the material and (b) as a function of depth

Figure 4: Ratio of the optical absorption rate per in as (a) function of absorption coefficient at different for photons with different absorption coefficients.

Figure 4 (a) illustrates that; photons with different energies have different absorption rates at a given depth. When a point at depth $x$ below the top surface of a given semiconductor is illuminated by photons of different energy (or different absorption coefficients, $\alpha$ ), the absorption rates for photons with very low and very high energies are negligibly very small. However, a photon with penetration depth equal to $1 / \alpha$ has a maximum absorption rate $\alpha \Phi_{0}(1-\mathrm{R}) \exp (-1)$ at that particular point as compared to other photons with energies less than or greater than its energy. That means, a hill of absorption rate of height $\alpha \Phi_{0}(1-\mathrm{R}) \exp (-1)$ is formed around the $\alpha=1 / x$ by the photons with different energies as shown Figure 4 (a).

One can also see from Figure 4 (a) and (b) that, the absorption rate lost due to reflection at the illuminated surface is $\alpha \Phi_{0} R$ and hence all photons have maximum absorption rate of $\alpha \Phi_{0}(1-\mathrm{R})$ just under the illuminated surface of the semiconductor. At the penetration depth of each photon, the absorption rate falls to $\alpha \Phi_{0}(1-\mathrm{R}) \exp (-1)$ or $37 \%$ of the maximum absorption rate at the surface. Figure 4 (b) also clearly depicts that, high energy photons are highly absorbed near the surface and low energy photons are penetrating into the deep bulk without being absorbed (or with less absorption).

\subsection{Doping level dependence of photo-response at the under the surface and in the deep bulk}

Figure 5 shows the room temperature (300K) doping level dependence of the excess minority carrier lifetime (a) just under the illuminated surface and (b) in the deep bulk of GaSb semiconductor. To analyze these results, first the pinning position for the surface Fermi level position is kept at $300 \mathrm{meV}$ regardless of the doping level reported by several authors [59]. Then, the position of the Bulk Fermi energy, $E_{B F}$ relative to the VBM is determined using relation (2-26) for all the samples with different doping levels.

The variation of the maximum energy band bending at the surface of each sample is also determined using 
relation (2-25) the radiative, Auger and effective excess minority carrier lifetimes are determined using Eqn. (220) along with Eqns. (2-50), (2-53) and (2-54) by considering only the doping level in the deep bulk and the variation of the majority carrier density with energy bending at different positions.

For non-degenerate semiconductors, the values of the band to band excess minority carrier lifetimes, $\tau_{R}, \tau_{A}$ and $\tau_{e f f}$ decrease with increasing the doping levels in the deep bulk as shown in Figure 5 (b) and remain constant irrespective of the doping level under the surface of the semiconductor as shown in Figure (a).
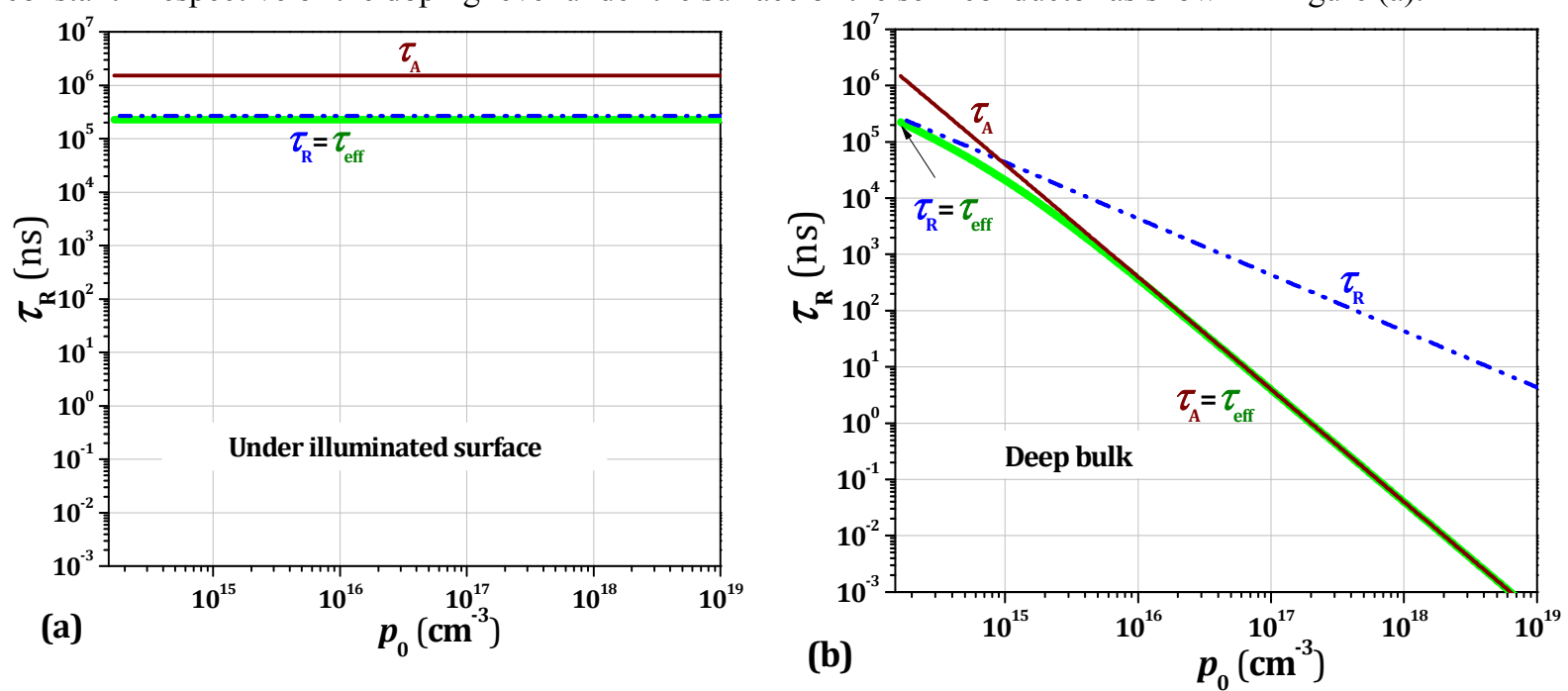

Figure 5: Doping level dependence of the excess minority carrier lifetime (a) under the illuminated surface and (b) in the deep bulk of GaSb semiconductor.

This is because in the bulk, the value of the majority carrier density, $p_{0}$ is directly related to the doping level and hence with increasing the doping level the value of $p_{0}$ increases and as a result the excess minority carrier lifetimes described by the relations (2-50), (2-53) and (2-54) decreases with increasing doping in the deep bulk of the semiconductor as shown Figure 5 (b). Nevertheless, the energy bending at the surface decreases and the values of the products on the right sides of Eqn. (2-20) and (2-21) remain constant with variations of the doping levels. Hence, the value of the majority carrier density, $p_{0 S}$ under the surface remains independent of the doping level. This makes the respective low level injection radiative, Auger and effective excess minority carrier lifetimes described by the relations (2-50), (2-53) and (2-54) to remain constant irrespective of the variation of the doping levels just under the surface of the semiconductor as depicted in Figure 5 (a). One can see from 5 (a) that, radiative recombination (Photon emission) dominates the under surface region for the entire doping regime. However, radiative recombination dominates only a small portion or the lowest level of the doping regime in the deep bulk and the Auger recombination (impact ionization) dominates most of the higher doping level. The difference between the excess carrier lifetimes at the under surface and the deep bulk increases with increasing the doping level. The results obtained for the variation of the excess carrier lifetimes with the doping level is in complete agreements with the reports of several authors $[60,61]$.

\subsection{Variation of photo-response with depth below the illuminated surface}

Figure 6 illustrates the position dependence of room temperature (a) energy band bending for samples with doping levels $10^{16} \mathrm{~cm}^{-3}, 10^{17} \mathrm{~cm}^{-3}, 10^{18} \mathrm{~cm}^{-3}$ and $10^{19} \mathrm{~cm}^{-3}$, (b) the respective low level injection radiative, Auger and effective lifetimes, $\tau_{R}, \tau_{A}$ and $\tau_{\text {eff }}$ excess minority carrier lifetimes for a sample with doping level $10^{17} \mathrm{~cm}^{-3}$ and (c) effective excess minority carrier lifetime, $\tau_{e f f}$ for samples used in (a). The variation of energy bending and the thermal equilibrium majority carrier density with the depth below the surface is described using relations (2-23) and (2-20), respectively. The width of the under surface depletion layer is also determined using relation (2-22). The radiative, Auger and effective excess minority carrier lifetimes are determined by considering the variation of the majority carrier with energy bending at different positions Eqn. (2-20) along with Eqns.(2-50), (2-53) and (2-54). 

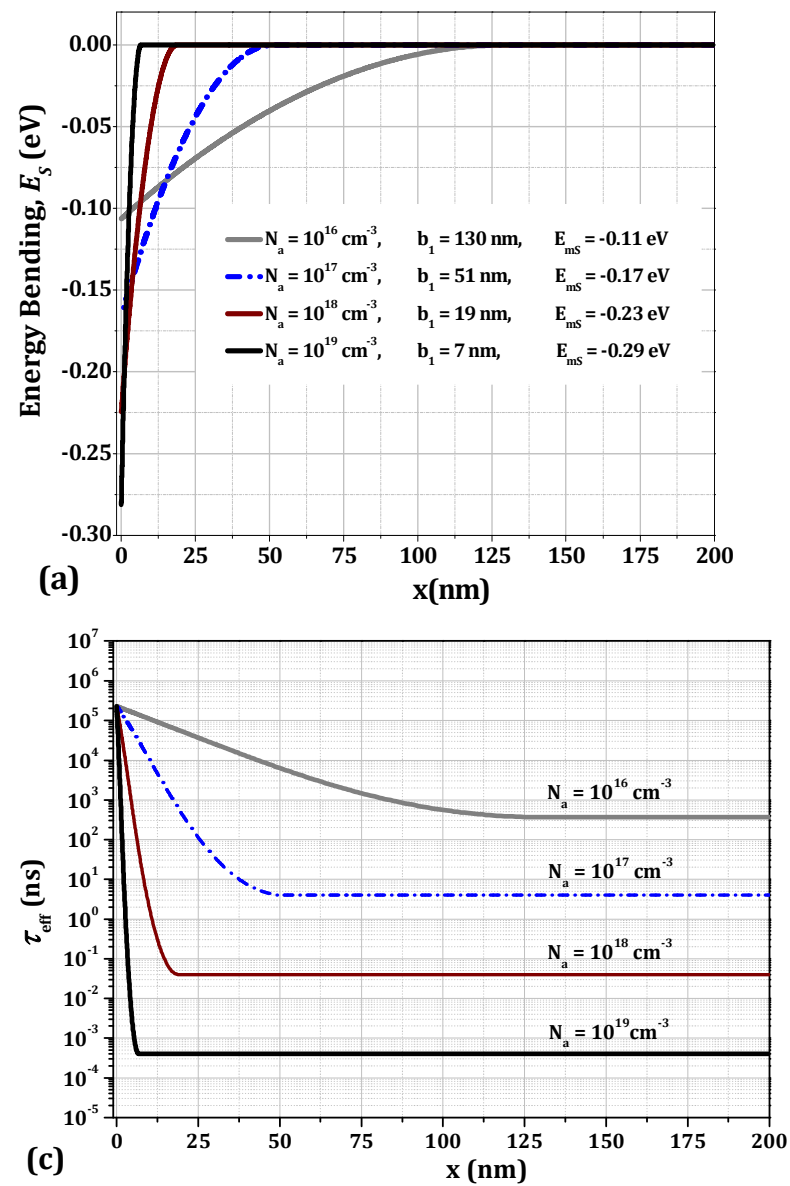

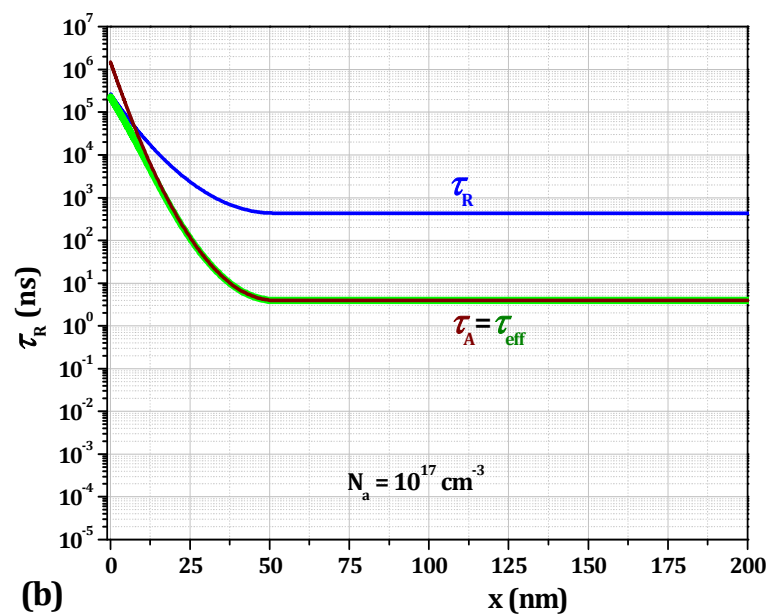

Figure 6: Position dependence of room temperature (a) energy band bending for samples with different doping levels, (b) the respective low level injection radiative, Auger and effective lifetimes, $\tau_{\mathrm{R}}, \tau_{\mathrm{A}}$ and $\tau_{\text {eff }}$ excess minority carrier lifetimes for a sample with doping level $10^{17} \mathrm{~cm}^{-3}$ and (c) effective excess minority carrier lifetime, $\tau_{\text {eff }}$ for samples considered in (a).

Since the style of the variation of the Auger excess minority carrier lifetime with the depth below the illuminated surface is the same as that of the variation of radiative excess minority carrier lifetime with the depth below the illuminated surface, except the difference in magnitude as depicted in Figure 6 (b), we use only the variation of radiative excess minority carrier lifetime in the analysis of the variation of excess minority carrier lifetime with the depth below the illuminated surface for samples with different doping levels shown in Figure 6 (c).

For non-degenerate semiconductors, the energy bending at the surface increases with increasing the doping levels, but, the width of the under surface depletion layer decreases with increasing the doping levels. The values of the band to band effective excess minority carrier lifetimes, $\tau_{R}, \tau_{A}$ and $\tau_{\text {eff }}$ remain fixed irrespective of the doping level just under the surface, decreases with increasing the depth below the surface in the depletion layer and decreases with increasing the doping levels in the bulk of the material and as illustrated in Figure 6 (c). The reason for the variation of the effective excess minority carrier lifetimes under the surface and the deep bulk is discussed in detail in section 4-3 above. Figure 6 (b) also shows that, the radiative recombination rate dominates the region closer to the surface and the Auger recombination rate dominates the most portion of the depletion layer including the deep bulk.

\subsection{Combined effects of doping and illumination energy on the Photo-response of entire bulk}

Figure 7: illustrates the room temperature low injection level radiative excess carrier lifetime at different locations below the illuminated surface (a) as function of majority carrier concentration and (b) as function of absorption coefficient for a sample with doping level of $10^{18} \mathrm{~cm}^{-3}$. Since the radiative excess carrier lifetime is very suitable for the effects illumination energy on the photo-response of the material, the analysis for this result is performed by using only radiative recombination mechanism. As can be seen from Figure 7 (a), the low injection level radiative excess carrier lifetime remains constant irrespective of doping just under the surface as already discussed in section 4-3 previously. For a point situated at depth $x$ below the surface in the depletion region, the excess minority carrier lifetime at the point rapidly decreases with increasing the doping level until the doping level reaches the maximum level that this point becomes the border of the depletion layer; $x=b_{1}$. The point of $x=b_{1}$ is the maximum common point of the curve of $x$ and the bulk line shown at the bottom of Figure 7 (a). When the doping level exceeds this level, the point under consideration becomes part of the deep bulk, $x>b_{1}$ and the excess 
carrier lifetime at the point decreases slowly in similar manner as the doping level dependence of the excess carrier lifetime in the deep bulk shown in Figure 7 (b).
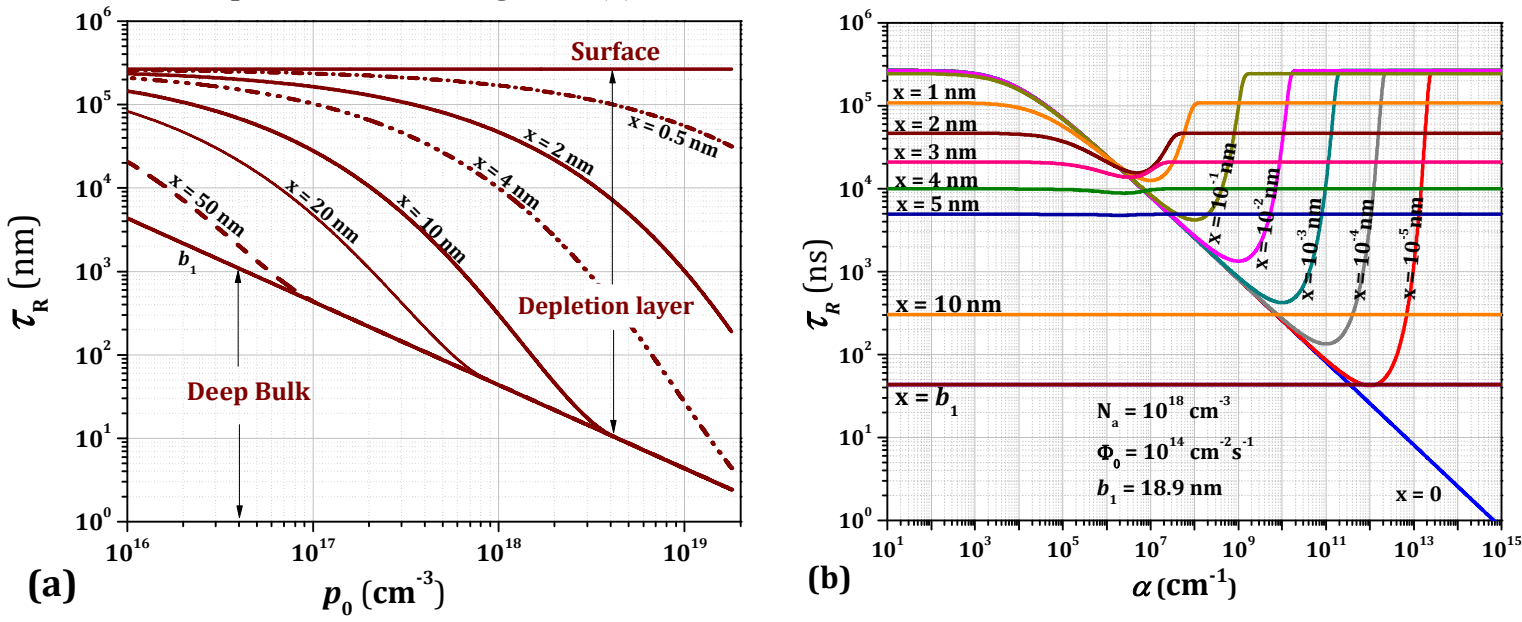

Figure 7: Room temperature low injection level radiative excess carrier lifetime at different locations below the illuminated surface (a) as function of majority carrier concentration and (b) as function of absorption coefficient for a sample with doping level of $10^{18} \mathrm{~cm}^{-3}$.

This bottom line in Figure 7(a) corresponds to the broken blue line of the doping level dependence of the radiative excess carrier lifetime in the deep bulk shown in Figure 7(b) above. Since $b_{1}$ has inverse relationship with the doping level, $N_{a}$, the point of $x=b_{1}$ is shifted to the low doping regime with increasing $x$ as illustrated by the maximum common points of the curve of $x$ and the bulk line shown at the bottom of Figure 7 (a). This figure also clearly shows that, the gap between the photo-responses of the under surface and the deep bulk increases with increasing the doping level.

Next, we wish to investigate the effects of the energy of the illumination at different positions in a given sample of semiconductor. To do so, the doping is adjusted to high level, $10^{18} \mathrm{~cm}^{-3}$, the injection level made to be slightly low, $10^{14} \mathrm{~cm}^{-2} \mathrm{~s}^{-1}$ and the absorption coefficient is varied from very low to very high values as depicted in Figure 7 (b). For a point located just under the surface, the excess carrier lifetime remains at its maximum constant value (equal to the excess carrier lifetime shown by broken blue line in Figure 6 (a)) at very low energy regime and decreases with increasing the absorption depth as depicted by the $x=0$ slanted blue line in Figure 7(a). As the depth, $x$ increases more, the excess carrier lifetime remains at its maximum constant value as in the under surface $x=0$ region, reaches its minimum value at the penetration depth of the illumination and again reaches the maximum value at the surface in the high energy regimes. The valleys of excess carrier lifetimes formed at the penetration depths between the two plateaus at very low and very high energy regimes is related to the inverse of the hills of absorption rates discussed in Figure 4 (a). The position of this deep valley shifts to the low energy regime and its depth also decreases with a slight increase in the depth $x$ below the illuminated surface as shown by curves $x=10^{-5} \mathrm{~nm}, 10^{-4} \mathrm{~nm}, 10^{-3} \mathrm{~nm}, 10^{-2} \mathrm{~nm}$ and $10^{-1} \mathrm{~nm}$ in Figure 7(b). As $x$ approaches the depletion layer width, $b_{1}$, the depth of the valley of the excess carrier lifetime diminishes to zero and the excess carrier lifetime decreases uniformly as depicted illustrated by the curves of $x=1 \mathrm{~nm}$ to $18.9 \mathrm{~nm}$ (or $b_{1}$ ) in Figure 7( (b). Finally, for $x \geq b_{1}$, the excess carrier lifetimes remains at its minimum constant value (zero energy bending) as shown by the lower brown horizontal line represented by $x=b_{1}$ in Figure 7(b), the results obtained for the variation of the excess carrier lifetimes with the absorption coefficients and depth is in complete agreements with the reports of several authors $[11,62,63]$.

\subsection{Injection level dependence of excess carrier lifetime}

In general, photo-generated carriers can be recombined both with opposite photo-generated carriers and thermallygenerated carriers in the material. Each of the recombination rates for the photo-generated carriers discussed above is hence the sum of the recombination rate of a photo-generated carrier with opposite photo-generated carriers and thermally-generated carriers. The net recombination rate of photo-generated carriers with other photo-generated carriers is dominant in high injection level material, while the net recombination rate of a photo-generated carrier with the thermally generated carriers is dominant in low injection level material. As a result, the excess carrier life time given in Eqns. (2-46), (2-51) and (2-54) remain only a function of photo-generated carrier concentrations in high injection level system and only a function of thermally-generated carrier concentrations in low injection level system.

The description of the injection level dependence of the excess carrier lifetimes can be predicted by varying the photo-generated carriers in relations (2-46), (2-51) and (2-54). Figure 8 illustrates the injection level 
dependence of room temperature excess minority carrier lifetime in GaSb samples (a) under the illuminated surface from intrinsic level to doping level of $10^{19} \mathrm{~cm}^{-3}$; and in the deep bulk at (b) intrinsic level, (c) doping level of $10^{17}$ $\mathrm{cm}^{-3}$ and (d) doping level of $10^{19} \mathrm{~cm}^{-3}$. Due to the surface Fermi pinning position, the region under the surface attains a minimum constant majority carrier density given by relation (2-24) which is independent of the doping level of the material as discussed in section 4-2 above. This makes the under surface region excess carrier life time to remain constant in the low injection level region $\left(p_{0 S}<\delta n\right)$ for the entire doping regime as shown in Figure 8(a). The under surface radiative excess carrier lifetime remains dominant at low injection level $\left(p_{0 S}<\delta n\right)$ irrespective of the doping level. The under surface Auger excess carrier lifetime is also dominant at high injection level $\left(p_{0 S}>\delta n\right)$ irrespective of the doping level.
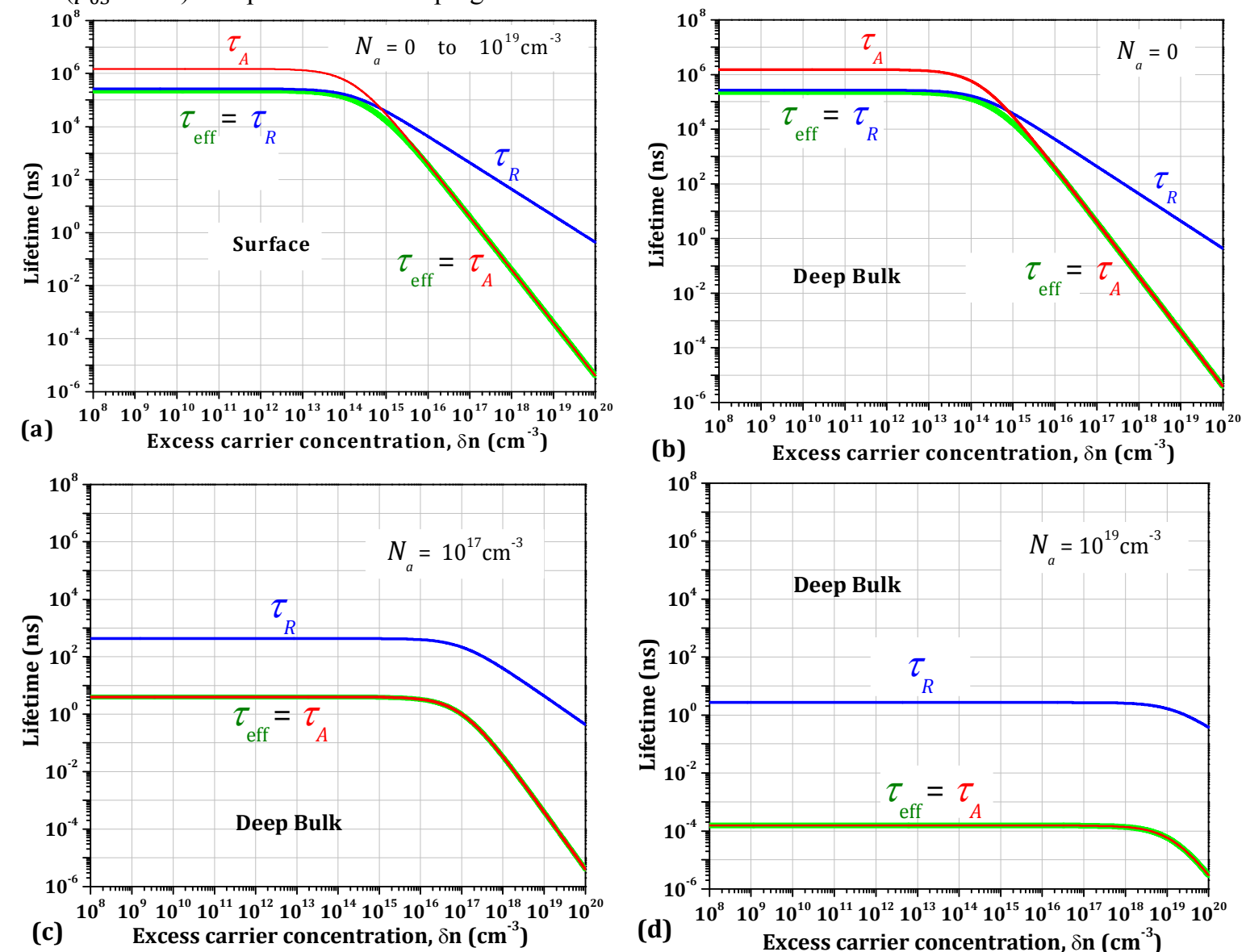

Figure 8: Injection level dependence of room temperature excess minority carrier lifetime in GaSb samples (a) under the illuminated surface from intrinsic level to doping level of $10^{19} \mathrm{~cm}^{-3}$; and in the deep bulk at (b) intrinsic level, (c) doping level of $10^{17} \mathrm{~cm}^{-3}$ and (d) doping level of $10^{19} \mathrm{~cm}^{-3}$.

In Figure 8(c) and (d), both the radiative and the Auger excess carrier lifetimes in the deep bulk remain constant at low injection level $\left(\delta n<p_{0}\right)$ and decreases in the high injection regime $\left(\delta n>p_{0}\right)$. The radiative excess carrier lifetime in the deep bulk can be dominant at low injection level in a sample of relatively very low donor concentration (Figure 8 (b)). The photo-responses of the deep bulk and the under surface region is similar at intrinsic level as depicted in Figure 8(a), (b). The deep bulk Auger excess carrier lifetime is dominant at low injection level in a sample of relatively high donor concentration and dominant at high injection level in all the samples in the high injection level samples irrespective of the donor concentrations as shown in Figure 8 (b), (c) and (d). This is because of most of the photo-generated carriers are involved in the process of generating electronhole pairs (impact ionization) rather than giving off photons during their recombination with the opposite charge carriers. The results obtained for the variation of the excess carrier lifetimes with the injection level is in complete agreements with the reports of several authors $[64,65]$.

\section{Conclusions}

In this study, the effects of the energies of the illuminations on the photon absorption rates at different locations below the illuminated surface inside GaSb sample are described. Then, the effects of doping, energy of illumination and injection level of photon flux density on the room temperature photo-responses of GaSb at different locations below the illuminated surface is investigated. 
The results obtained from the description of photon absorption rate, shows that; photons with different energies have different absorption rates at a given depth. At depth $x$ below the illuminated surface, photons with depths less and greater than the penetration depths of the illuminations have negligibly small absorption rates, so that the absorption rate forms a hill of maximum height equal $37 \%$ its value at the surface. The absorption rate occurs at the penetration depth of a photon is equal to $\alpha \Phi_{0}(1-\mathrm{R}) \exp (-1)$ or $37 \%$ of the maximum absorption rate at the surface. High energy photons are highly absorbed near the surface and low energy photons are penetrating into the deep bulk without being absorbed (or with less absorption). The analysis of the photo responses of GaSb indicates that, the energy bending at the surface increases with increasing the doping level, while the width of the deletion layer decreases with doping level. The values of the band to band excess minority carrier lifetimes, decrease with increasing the doping levels in the deep bulk and remain constant irrespective of the doping level under the surface of non-degenerate semiconductor. Radiative recombination dominates the under surface region for the entire doping regime, while it dominates only a small portion or the lowest level of the doping regime in the deep bulk. The Auger recombination dominates most of the higher doping level in the deep bulk regions.

Excess minority carrier lifetime decreases with increasing the depth below the surface in the depletion layer and remains constant in at all positions in the deep bulk of doped semiconductor. For points situated in the depletion region, the low injection level excess minority carrier lifetimes decrease have relatively very high value and rapidly with increasing the doping levels as compared to the corresponding excess minority carrier lifetimes in the deep bulk. The excess carrier lifetime at the under surface region of a highly doped semiconductor at relatively low injection level semiconductor remains constant and has very high value in the low illumination energy regime and diminishes to zero with increasing the energy of the illumination. For points situated deep inside the depletion layer, the excess carrier lifetime in the same material has very deep valley of minimum point at the penetration depth $(1 / \alpha)$ surrounded by plateaus of the same height on either sides of the low and high illumination energy regimes. The position of this deep valley shifts to the low energy regime and its depth also decreases with a slight increase in the depth $x$ below the illuminated surface. As $x$ approaches the depletion layer width, $b_{1}$, the depth of the valley of the excess carrier lifetime diminishes to zero and the excess carrier lifetime decreases uniformly until it reaches the corresponding value in the deep bulk. This shows that, the photo response of the under surface layer is suppressed by high energy illuminations and the photo-responses of the points situated deep in the depletion layer is suppressed by photons that have equal penetration depths equal to the depths of the corresponding points. Points located in the low injection level deep bulk are not affected by the energies of the illuminations.

The under surface region excess carrier lifetime remains constant in the low injection level region for the entire doping regime. The under surface radiative excess carrier lifetime remains dominant at low injection level irrespective of the doping level. The under surface Auger excess carrier lifetime is also dominant at high injection level irrespective of the doping levels. The radiative excess carrier lifetime in the deep bulk can be dominant at low injection level in a sample of relatively very low donor concentration. Hence, the photo-responses of the deep bulk and the under surface region is similar at intrinsic level. The deep bulk Auger excess carrier lifetime is dominant at low injection level in a sample of relatively high donor concentration and dominant at high injection level in all the samples irrespective of the donor concentrations. This is impact ionization is the dominant mechanism in samples with very high carrier concentrations (thermal or photo generated) than optical generation of carriers.

\section{References}

[1] G.L Pearson and W. H. Brattain, "History of semiconductor research," Pre-1900 Semiconductor Research and Semiconductor Device, Vols. 43, pp. 1794-1806, 1955.

[2] Faraday, M, "Experimental Researches in Electricity ,Bernand Quaritch,London," vol. I, pp. 122-124, 1839.

[3] G. Calzaferri, D. Brühwiler, S. Glaus, D. Schürch, A. Currao, and C. Leiggener "Quantum-Sized Silver, Silver Chloride and Silver Sulfide Clusters," Journal of ImagingScience and Technology,, vol. 45, July/August 2001.

[4] Weinheim: Wiley, Host-Guest-Systems Based on Nanoporous Crystals, F. S. U. S. a. M. W. F. Laeri, Ed., pp. 435-436 2003.

[5] Mehta,V.K, Principles of Electronics, 6 December 2015 ed.,p.56,01-01- 2008.

[6] Elizabeth A.Jones, Semiconductors physics and devices(PDF).

[7] E. M. O. L. G. Johonson,"Energy Band in Semiconductors," applied phys., pp. 3-50,2011.

[8] Carl W.Wilmsen, Physics and Chemistry of III-V Compound Semiconductors interfaces, pp 339-342.

[9] P. C. R.K. Lal, A comparison of dominant recombination mechanisms in n-type InAsSb materials, India,Banaras Hindu University, 2006.

[10] J. B. M.W. Shura V. Wagener, Physica B 407, Photoconductivity spectroscopy of p-type GaSb thiln film, 2012.

[11] D. K. Schroder, "The concept of generation and recombination lifetimes in semiconductors" ,IEEE Trans. 
Electron Devices,pp 1336-1338,vol. ED-29, Aug. 1982.

[12] Divij Bhatia, "Study of sumiconductor near surface region using photo conductive technique" Pennsylvania State University 2011.

[13] J. F. Roller, Contactless Spectral-Dependent Measurement of Bulk Lifetime and Surface Recombination Velocity in Silicon Photovoltaic Materials, University of Maryland, 2016.

[14] F. B. L. H. J. Bardeen, "Indirect transitions from the valance to the conduction bands, photoconductivity conference" J.phys, pp. 146-154, 1954.

[15] M. e. a. Buscema, "Large and tunable photo thermoelectric effect in single-layer MoS2. Nano. Lett., 2013".

[16] [ Dare-Edwards, M. P., Hammett, A. \& Goodenough, J. B, "The Efficiency of Photo generation of Hydrogen at p-Type III/V Semiconductor," Journal of Electroanalytical Chemistry, Vols. 119, pp. 109-123, (1981).

[17] Varshni Y. P., "Temperature dependence of the energy gap in semiconductors" Physica, Vols. 34, p. 149, (1967).

[18] L.G. Ferguson and L.M. Fraas, "Theoretical Study of GaSb PV Cell Efficiency as a Functionof Temperature', Solar Energy Materials \& Solar Cels" Performance evaluation of a GaSb thermo-photovoltaic converter, vol. 39, no. $\mathrm{N}^{\circ} 1$, pp. 11-18, 1995.

[19] J. Callaway, Energy Band Theory,Academic Press, New York, (1964).

[20] M. J. Kelly, Physics and Technology of Submicron Structures (G. Bauer, F. Kuchar,and H. Heirich, eds.), Berlin, Springer-Verlag, p. 174-196,(1987).

[21] I. A. Y.Lubiaiker, "direct band and indirect band semiconductors Depend on temperure in physics" p. 38, 1995.

[22] L.L. Li, W. Xu, Z. Zeng, A.R. Wright, C. Zhang, J. Zhang, Y.L. Shi and T.C. Lu, "Mid Infrared Absorption by Short-Period InAs/GaSb type II superlattices" Microelectronics, Vols. 40, no. №4-5, pp. 815 - 817, 2009.

[23] E. Rosencher and B. Vinter, "Opto-electronic, Thomson-CSF Masson" no., 1998.

[24] IoffePhysico-TechnicalInstitute, "http://www.ioffe.rssi.ru/SVA/NSM/Semicond/GaSb/bandstr.html," [Online].

[25] Sze, S. M., Physics of Semiconductor Devices [a comprehensive advanced text], Wiley, New York), (1981). [26] Yu, P. Y. and M.Cardon, Fundamentals of Semiconductors: Physics and Materials Properties, (Springer, New York), (1996).

[27] Dutoit, E. c., Cardon, F., Gomes, W. P, " Ber. Bunsenges. Phys. Chem." vol. 80, no. 1, pp. 475-81, 1976.

[28] F. Pierret, "Advanced Semiconductor Fundamentals, in Modular series on solid state devices" vol. 6, 1987.

[29] A. A. Mermin, "Solid State physics" in sounders,Philodelphia, pp. 76-81, 1976.

[30] Blood, P. and J. W. Orton, The Electrical Characterization of Semiconductors: Majority Carriers and electron ststes, Landon: Accadamic press, (1992).

[31] G. Stollwerk , A .W . Bett, S. Keser, and O.V .Sulima, " GaAs/GaSb Tandem Concentrator solar cell in proc.14TH European PVSEC. spain,Barcelona, pp. 993-998,1997.

[32] Sze, S. M. (Ed.), Modern Semiconductor Device Physics, Wiley, New York, (1998).

[33] Tyagi, M. S., Introduction to Semiconductor Materials and Devices, Wiley, New York, (1991).

[34] Wang, S., Fundamentals of Semiconductor Theory and Device Physics (Prentice-Hall, Englewood), (1989) .

[35] S.K. Ghandhi, Semiconductor Power Devices, Wiley, New York, 1977.

[36] J. J. Sparkes, , Semiconductor Devices, Second edition, London, (CHAPMAN \& HALL), ( 1994).

[37] A. Many, Y. Goldstein, N.B. Grover,"Semiconductor Surfaces, 2nd ed.,North-Holland,Amsterdam" NorthHolland, Amsterdam, 1971.

[38] W. Mo Ėnch," Semiconductor Surfaces and Interfaces." Berlin,Springer, 1993.

[39] H. Lu Èth, Surfaces and Interfaces of Solids, 2nd ed., Berlin,Springer, 1993.

[40] D.J. Fitzgerald and A.S. Grove,"Surface Recombination in Semiconductors," Surface Science, Vols. 9, no. 1, p. 347-369, Feb. 1968.

[41] Ning An, Guojun Liu, ${ }^{*}$ Zhipeng Wei, ${ }^{* *}$ Rui Deng, Xuan Fang, Xian Gao, Yonggang Zou, Mei Li, Xiaohui Ma, "Study on Neutral Sulphur Passivation of Gallium Antimonide Surface" in National Key Laboratory of High Power Semiconductor Lasers University of Science and Technology, Changchun, 23-25,2012.

[42] Mattias Jeppsson, Kimberly Dick, Henrik.Nilsson,etc, "Characterization of GaSb nanowires grown by MOVPE" Journal of Crystal Growth, vol. , p. 5119-5122, 2008.

[43] Thomann, I., Pinaud, B. A., Chen, Z., Clemens, B. M., Jaramillo, T. F.,Brongersma, M. L., "Nano Lett.," vol. 11, p. 3440, (2011).

[44] R. H. Bube, Photoconductivity of Solids, Wiley, New York, 1960.

[45] Pankove, J. I. , Optical Processes in Semiconductors. Englewood Cliffs, NJ: PrenticeHall, 1971.

[46] Wolfe, C. M., G. E. Stillman, and N. Holonyak, Jr. Physical Properties of Semicon-ductors. Englewood Cliffs, NJ: Prentice Hall, 1989.

[47] M. E. Law, E. Solley, M. Liang, and D. E. Burk, "IEEE Elec. Dev. Lett. " Vols. 12, p. 40, (1991).

[48] S. S. Li, "Appl. Phys. Lett., vol. 29, p. 126, 1976. 
[49] D. V. Lang, " J. Appl. Phys. "vol. 45, p. 3014-3022, (1974).

[50] S. S. Li, W. L. Wang, P. W. Lai, and R. Y. Loo, "Recombination Mechanisms" IEEE Trans. Electron devices, Vols. ED-27, no. 3, p. 857, (1980).

[51] P. T. Landsberg and A. F. W. Willoughby (eds.)"on Recombination Mechanisms in Semiconductors,in Solid State Electronics 21" in Proceeding of the International Conference, (1978).

[52] R. J. Kumar, J. M. Borrego, P. S. Dutta and R. J. Gutmann"Auger and Radiative Recombination Coefficients in $0.55 \mathrm{eV}$ InGaAsSb" Lexington, 2004.

[53] Yeasir Arafat*, Farseem M. Mohammedy, M. M. Shahidul Hassan, "Optical and Other Measurement Techniques of Carrier Lifetime in Semiconductors" Journal of International Optoelectronic Engineering , vol. 2, no. 2, pp. 5-11, 2012 .

[54] Anikeev et al., "Measurement of the Auger recombination rate in p-type $0.54 \mathrm{eV}$ GaInAsSb by time-resolved photoluminescence" APPLIED PHYSICS LETTERS, vol. 83, p. 16, 20 OCTOBER 2003.

[55] V. Benda, J. Gowar, D.A. Grant, Power Semiconductor Devices: Theory and Applications, Chichester, Wiley, 1999.

[56] P. T. Landsberg, Solid State Physics in Electronics and Telecommunications, London ,Academic Press, (1960).

[57] A. Many and R. Bray, "Lifetime of Excess Carriers in Semiconductors" in Semiconductors, London , Heywood and Co., pp.117-151, (1958) .

[58] S. Rein, Lifetime spectroscopy: A method of defect characterization in silicon for photovoltaic applications, Berlin, Germany, Springer 2004 .

[59] D. R. Khanal, W. Walukiewicz, J. Grandal, E. Calleja and J. Wu, "determining surface fermi level pinning postion of InN nanowiresusing electrolyte geting" APPLIED PHYSICS LETTERS, vol. 95, pp. 173114-1, 2009. [60] A.N. Titkov, G.V. Benemanskaya, and G.N. Iluridze, "A.N. Titkov, Scale time for interband Auger recombination involving aspin-orbit-split valance band p-type GaSb crystals"Apply phys.lettrs, 1981.

[61] Ricardo Ascazubi, The Emission Spectroscopy of Narrow band-gap Semiconductors, New York, 2005.

[62] H. Nagel, C. Berge, and A.G. Aberle, "Generalized Analysis, of Quasi-Steady-State and Quasi-Transient Measurements of Carrier Lifetimes in Semiconductors" J. Appl. Phys, vol. 86, no. 1, pp. 6218-6221,Dec. 1999.

[63] S. K. Pang and A. Rohatgi, "Record high recombination lifetime in oxidized magnetic Czochralski silicon" Appl. Phys. Lett., vol. 59, no. 2, p. 195-197, July 1991.

[64] Mohammad ZiaurRahman, "Modeling Minority Carrier Recombination Lifetime in Silicon Solar Cell" International Journal of Renewable Energy Research, vol. 2, no. 1, pp. 449- 454, 2012.

[65] M.W.Shura,V.Wagener,J.R.Botha and M.C.Wagener, "Non-linear injection level dependence of the excess carrier lifetime of p-type GaSb thin films: A two-layer model" Journal of Applied Physics , vol. 111, p. 113104, (2012). 